\title{
Postprocessor and Macro Program for Calculation of the Displacements of the Working Coordinate Systems when Working by the Method $3+2$ axes
}

\author{
Tsvetan Kaldashev \\ Technical university of Sofia \\ Sofia, Bulgaria \\ kaldashev.cvetan@abv.bg
}

\begin{abstract}
This article discusses the possibility of developing a postprocessor and macro program for automatic calculation and input of the displacements in the working coordinate systems when working by the method 3 +2 axes. The ability to take the information from the CL Data file and its subsequent use by a macro program for certain calculations is used. For this purpose, a generalized G-POST postprocessor and a specialized FIL language are used.
\end{abstract}

Keywords - CNC, 3+2 axis, postprocessor, Euler angles

\section{INTRODUCTION}

Often, machining of prismatic body parts on five axial machines requires machining in several different positions. In this case the method is $3+2$ axes, where the two circular axes are used only for adjusting the rotation of the workpiece in the respective machined position. This method has the advantage that the workpiece is processed in one set, which leads to a reduction of the establishment error, and in some cases to compensate for systematic errors [3]. The working coordinate systems used for machining by the $3+2$ axis method can be set by using a separate working coordinate system G54-G59 for CNC Fanuc and others for each of the machined positions or additional ones - G154 P1-P99 for CNC Haas [1], G54.1 P1-P300 for CNC Mazak [2] or setting using command G68.2 to establish an inclined working plane. In this case a local coordinate system is created, where the values of displacement and rotation of the newly obtained coordinate system are set programmatically with command G68.2, i.e. there is no need to enter an offset in the Offset page of the machine. These various methods of adjustment may be applied as by treatment with machining, and in the methods associated with ball burnishing [5].

\section{DEVELOPING A POSTPROCESSOR AND MAKROPROGRAMA}

Each CNC machine is characterized by the machine coordinate system $\mathrm{O}_{\mathrm{M}}\left(\mathrm{X}_{\mathrm{M}}, \mathrm{Y}_{\mathrm{M}}, \mathrm{Z}_{\mathrm{M}}\right)$ (fig. 1) against which the workpieces are established and the coordinate system $\mathrm{O}_{\mathrm{T}}\left(\mathrm{X}_{\mathrm{T}}, \mathrm{Y}_{\mathrm{T}}, \mathrm{Z}_{\mathrm{T}}\right)$, towards which the cutting tool is oriented.

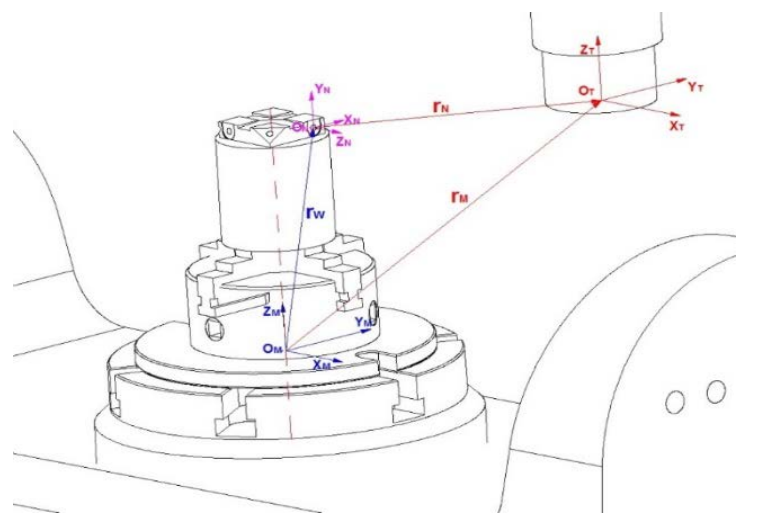

Fig. 1. Relationship between $\mathrm{O}_{\mathrm{M}}$ and $\mathrm{O}_{\mathrm{T}}$.

When moving the tool according to a program, in order to be able to achieve the dimensions according to the drawing between these two coordinate systems $\mathrm{O}_{\mathrm{M}}$ and $\mathrm{O}_{\mathrm{T}}$, it is necessary to make a dimensional connection. As a result of the attestation of the machine, the components of the vector $r_{M}$ connecting the coordinate system of the machine $\mathrm{O}_{\mathrm{M}}$ and the coordinate system of the tool $\mathrm{O}_{\mathrm{T}}$ when the working bodies of the machine are at a reference point are determined. When processing a given surface, it is 
necessary to determine the components of the vector $\mathrm{r}_{\mathrm{W}}$ connecting the coordinate system of the machine with the working coordinate system $\mathrm{O}_{\mathrm{H}}\left(\mathrm{X}_{\mathrm{N}}, \mathrm{Y}_{\mathrm{N}}, \mathrm{Z}_{\mathrm{N}}\right)$ (fig. 1). The vector $r_{N}$ is calculated automatically by the CNC and represents the relative displacement to the $\mathrm{O}_{\mathrm{N}}$ coordinate system, where the length of the tool is included.

The treatment of surfaces and holes by the method $3+$ 2 axes necessitate adjustment of the working coordinate systems for each of the processed position i.e. to determine the components of vectors $\mathrm{r}_{\mathrm{w} 1}, \mathrm{r}_{\mathrm{w} 2}$, $\mathrm{r}_{\mathrm{Wn}}$ (fig. 2)

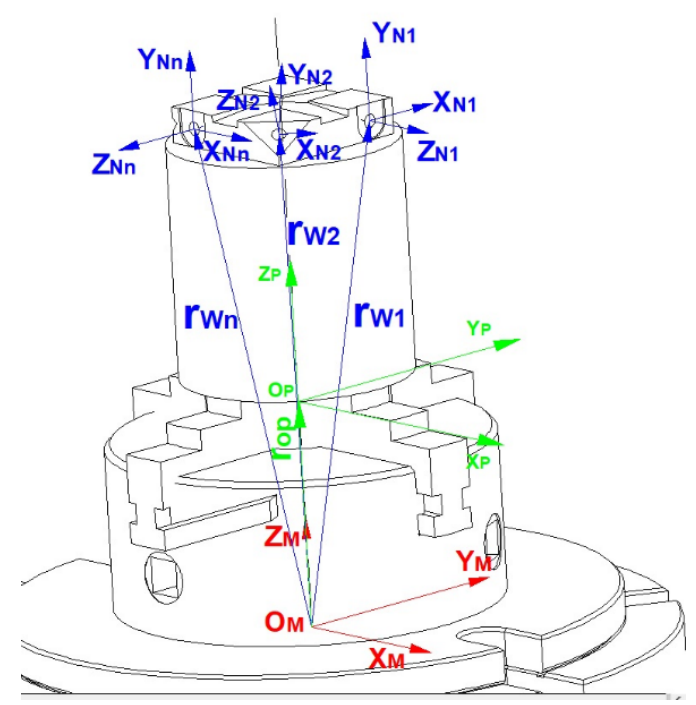

Fig. 2. Determination of the components of vectors $r_{W 1}, r_{W 2}, r_{W n}$

To determine the components of vectors $r_{W 1}, r_{W 2}, r_{W n}$, it is most convenient to use Euler angles. They determine the position of the different coordinate systems $\mathrm{O}_{\mathrm{N}}$ relative to the machine coordinate system $\mathrm{O}_{\mathrm{M}}$ by rotating sequentially about the three linear axes at an angle $\phi$ about the $\mathrm{X}$ axis, an angle $\theta$ around the newly obtained direction on the $\mathrm{Y}$ axis and an angle $\psi$ around the newly obtained position on the $\mathrm{Z}$ axis.

Macro program (using a subroutine), which performs calculation and automatically input the values of displacement of different coordinate systems working in Offset page by typing:

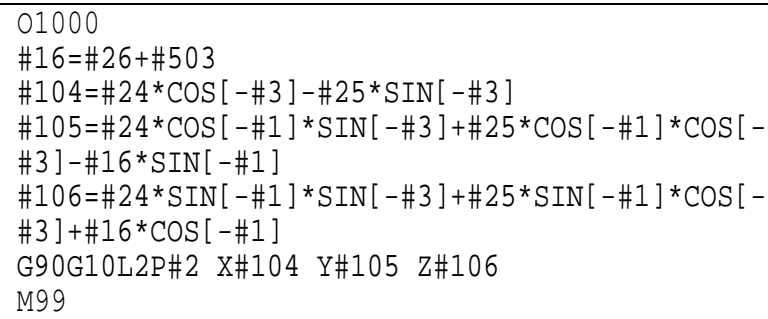

The values of variables \# 1, \# 2, \# 3, \# 24, \# 25, \# 26 are set with the command for simple reference to macro program G65 [3, 4] having the form:

G65 P... A... B... C... X... Y... Z...,

where the meaning of the individual arguments is: $\mathrm{P}$ number of the macro program; A - angle of rotation along the A axis; $\mathrm{B}$ - register of the coordinate system $\mathrm{G} 54, \ldots$, G59; $\mathrm{C}$ - angle of rotation along the $\mathrm{C}$ axis; $\mathrm{X}, \mathrm{Y}, \mathrm{Z}$ - the displacement along the $\mathrm{X}, \mathrm{Y}$ and $\mathrm{Z}$ axes of the working coordinate system with origin $\mathrm{O}_{\mathrm{N}}$ set with respect to $\mathrm{O}_{M}$

The new position of the working coordinate system is set with the sentence G90G10L2P\#2 X\#104 Y\#105 Z\#106, where L defines the category of displacement (L2 for displacement of coordinate systems), $\mathrm{P}$ - the register of the coordinate system in which enters the offset (the value is taken from argument B of command G65); X, Y and Z - the calculated offset value defined in variables \# 104 (for $X$ axis), \# 105 (for $Y$ axis) and \# 106 (for $Z$ axis) . Variable \# 503 sets the components of the vector rop, connecting the coordinate system of the machine $\mathrm{OM}$ and the reference base of the device $\mathrm{O}_{\mathrm{P}}\left(\mathrm{X}_{\mathrm{P}}, \mathrm{Y}_{\mathrm{P}}, \mathrm{Z}_{\mathrm{P}}\right)$ (fig. 2).

The developed postprocessor has the task to extract the values from the CL Data file and set them to arguments A, $\mathrm{B}, \mathrm{C}, \mathrm{X}, \mathrm{Y}$ and $\mathrm{Z}$ in command G65, which calls the macro program for calculation and introduces the offset of the used coordinate systems.

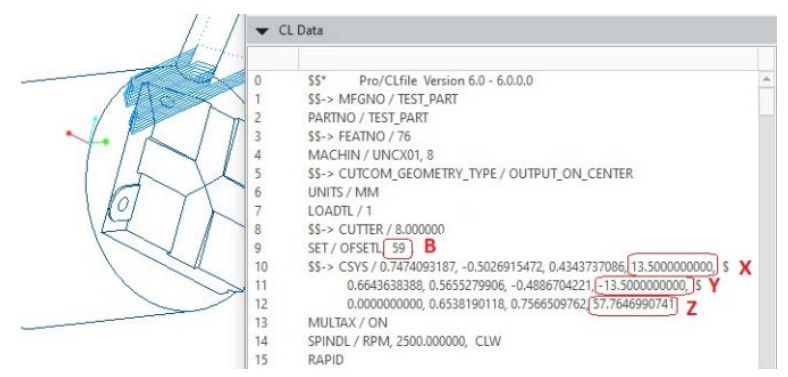

Fig. 3. Argument - value taken from CL Data file

In fig. 3 shows to which argument what value of the CL Data file is assigned. Using a specialized FIL language (Factory Interface Language) and a generalized G-POST postprocessor, I create an array of data about the coordinate systems used and their displacements relative to the reference base of the device. The RESERV command is used to create an array of data for further use. For example, RESERV / A, 4 will allow the following symbol-index combination A (1), A (2), A (3), A (4).

In order to be able to generate the G65 command line when generating the driver, it is necessary to use the Look ahead technique, which allows to read the entire CL Data file and extract the necessary information. In this case, a macro record having the structure is used:

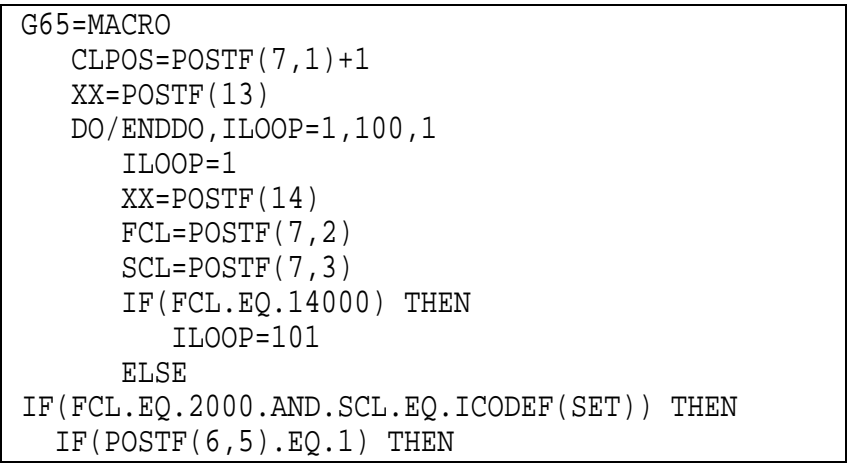




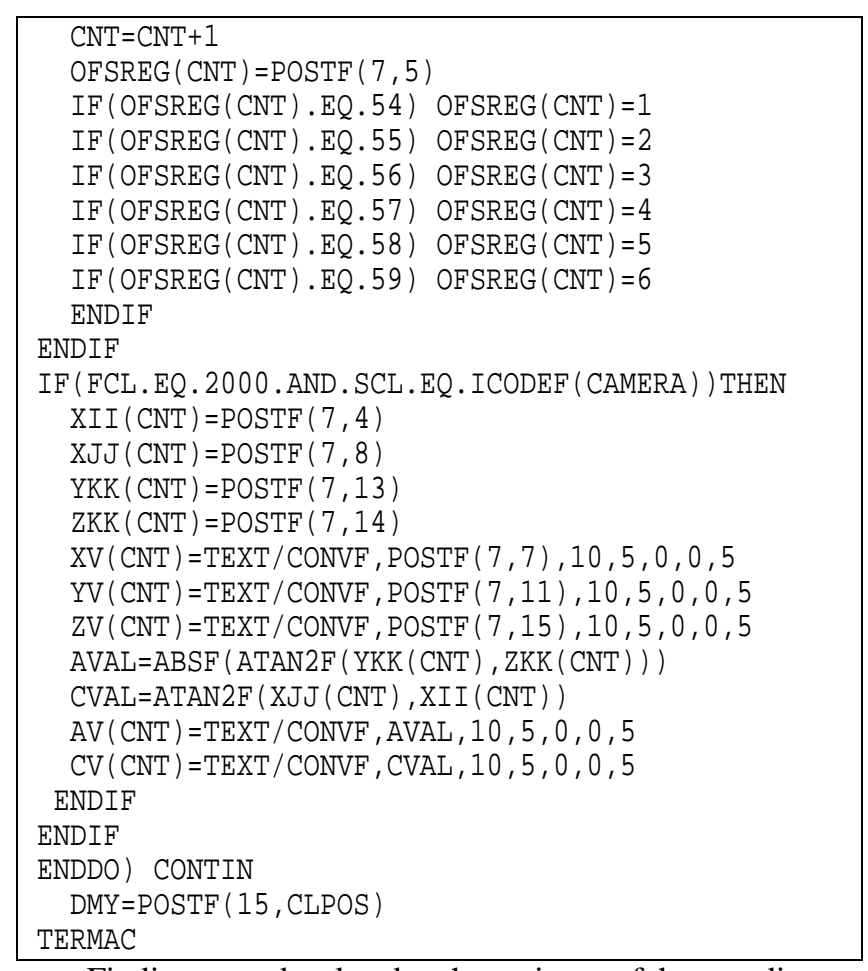

Finding records related to the registers of the coordinate system is performed with the operator IF (FCL.EQ.2000.AND.SCL.EQ.ICODEF (SET)) THEN, which creates an array of data for the used coordinate systems IF (OFSREG (CNT) ) .EQ.54) OFSREG $(\mathrm{CNT})=$ 1, IF (OFSREG (CNT) .EQ.55) OFSREG (CNT) = 2, etc. In a similar way, the displacement and angles of rotation of the coordinate systems relative to the machine coordinate system are determined, and together with this the data set is created. This part of the information is processed by the second IF operator (IF (FCL.EQ.2000.AND.SCL.EQ.ICODEF (CAMERA)) THEN).

The output of the created data set is performed at the beginning of the control program, in the records related to the machine. It has the structure:

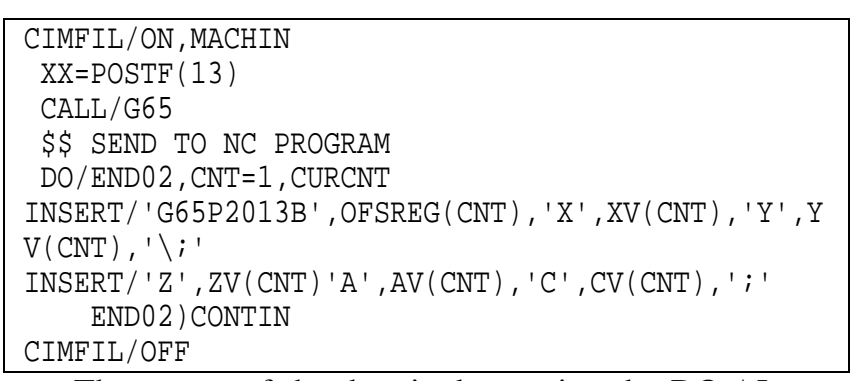

The output of the data is done using the DO / Loop operator, where the output is performed from the first index of the array $(\mathrm{CNT}=1)$ to the current one $(\mathrm{CURCNT})$. The output of the line with the G65 command is realized with the body of the DO / Loop cycle.

With the postprocessor developed in this way, a control program is generated (only a part of it is shown in fig. 4), for processing the workpiece shown in fig. 5 .

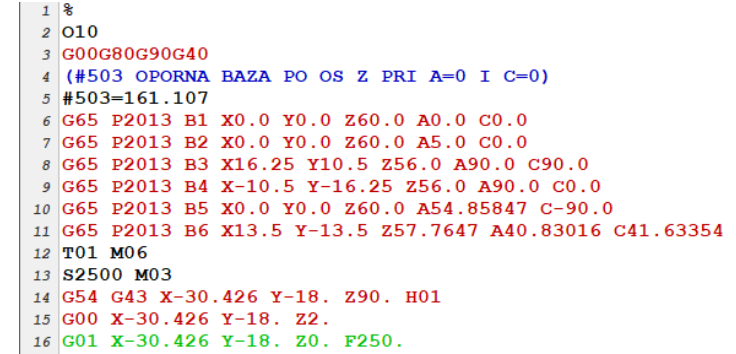

Fig. 4. Part of NC program

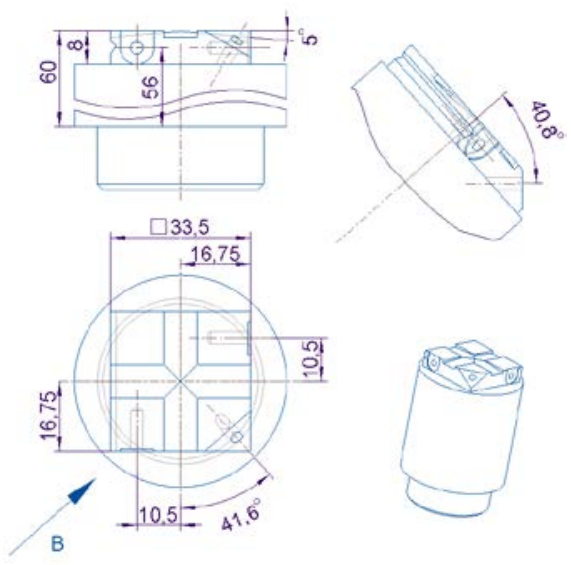

Fig. 5. Test workpiece

The postprocessor was checked - virtual with the use of Vericut and one on a machine with 5 axes and horizontal layout MC032 with CNC Fanuc 6MB and a test workpiece was processed (Fig. 5). In fig. 6 shows the machined workpiece on the machine.

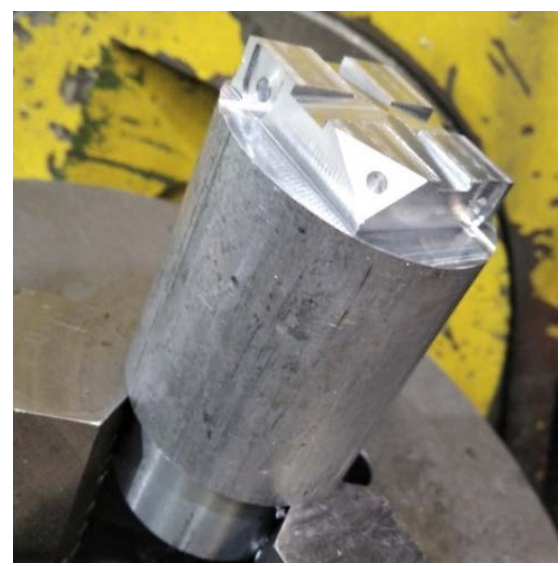

Fig. 6. Machined workpiecenon the machine

\section{CONCLUSION}

1. The developed postprocessor in combination with the macro program for automatic calculation and loading of the values of displacement of the working coordinate systems when working by the method $3+2$ axes is correctly working.

2. The proposed postprocessor and macro program are valid for machines with five axes in which the machine 
Kaldashev, Postprocessor and Macro Program for Automatic Calculation and Input of the Displacements of the Working Coordinate Systems When Working by the Method $3+2$ Axis

coordinate system coincides with the intersection of the two circular axes A and C.

\section{ACKNOWLEDGEMENTS}

The author would like to thank the Research and Development Sector at the Technical University of Sofia for the financial support.

\section{REFERENCES}

[1] Haas Mill Operator's manuals, Next Generation Control 96-8210 Revision F December 2017 English

[2] Mazatrol SmoothX Programming manuals, EIA/ISO program, 2019

[3] Hadjiiski P., Programming of CNC machine tools, Tehnical university of Sofia, 2010, ISBN 978-954-438-865-2A

[4] Ben Groves, Macro B programming manual

[5] Slavov, S., Dimitrov, D., Iliev, I., Variability of regular relief cells formed on complex functional surfaces by simultaneous five-axis ball burnishing, (2020) UPB Scientific Bulletin, Series D: Mechanical Engineering, 82 (3), pp. 195-206. 